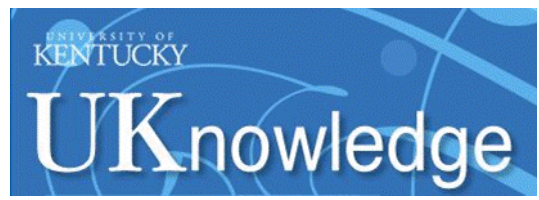

University of Kentucky

UKnowledge

$1-1-2018$

\title{
Objectively Measuring Effects of Electro-Acupuncture in Parkinsonian Rhesus Monkeys
}

\author{
Rui Zhang \\ University of Kentucky \\ Anders $\mathrm{H}$. Andersen \\ University of Kentucky, anders@uky.edu \\ Peter A. Hardy \\ University of Kentucky, peter.hardy@uky.edu \\ Eric Forman \\ University of Kentucky, esform2@uky.edu
}

April Evans

University of Kentucky

Follow this and additional works at: https://uknowledge.uky.edu/mrisc_facpub

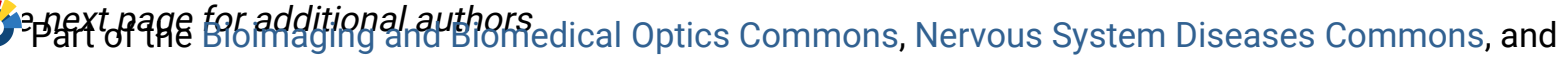
the Neuroscience and Neurobiology Commons

Right click to open a feedback form in a new tab to let us know how this document benefits you.

\section{Repository Citation}

Zhang, Rui; Andersen, Anders H.; Hardy, Peter A.; Forman, Eric; Evans, April; Ai, Yi; Yue, Jin; Yue, Guihua; Gash, Don M.; Grondin, Richard; and Zhang, Zhiming, "Objectively Measuring Effects of ElectroAcupuncture in Parkinsonian Rhesus Monkeys" (2018). Magnetic Resonance Imaging and Spectroscopy Center Faculty Publications. 3.

https://uknowledge.uky.edu/mrisc_facpub/3

This Article is brought to you for free and open access by the Magnetic Resonance Imaging and Spectroscopy at UKnowledge. It has been accepted for inclusion in Magnetic Resonance Imaging and Spectroscopy Center Faculty Publications by an authorized administrator of UKnowledge. For more information, please contact 


\section{Objectively Measuring Effects of Electro-Acupuncture in Parkinsonian Rhesus Monkeys}

\section{Digital Object Identifier (DOI)}

https://doi.org/10.1016/j.brainres.2017.10.006

Notes/Citation Information

Published in Brain Research, v. 1678, p. 12-19.

(c) 2017 Elsevier B.V. All rights reserved.

This manuscript version is made available under the CC-BY-NC-ND 4.0 license https://creativecommons.org/licenses/by-nc-nd/4.0/.

The document available for download is the author's post-peer-review final draft of the article.

\section{Authors}

Rui Zhang, Anders H. Andersen, Peter A. Hardy, Eric Forman, April Evans, Yi Ai, Jin Yue, Guihua Yue, Don M. Gash, Richard Grondin, and Zhiming Zhang 
Published in final edited form as:

Brain Res. 2018 January 01; 1678: 12-19. doi:10.1016/j.brainres.2017.10.006.

\title{
Objectively measuring effects of electro-acupuncture in parkinsonian rhesus monkeys
}

\author{
Rui Zhang ${ }^{\mathrm{a}, \mathrm{c}}$, Anders H. Andersen ${ }^{\mathrm{b}, c}$, Peter A. Hardy ${ }^{\mathrm{b}, c}$, Eric Forman ${ }^{\mathrm{c}}$, April Evans ${ }^{\mathrm{c}}, \mathbf{Y i ~ A i}^{\mathrm{c}}$, \\ Jin Yue ${ }^{d}$, Guihua Yue ${ }^{\mathrm{e}}$, Don M. Gash ${ }^{\mathrm{c}}$, Richard Grondin ${ }^{\mathrm{c}}$, and Zhiming Zhang ${ }^{\mathrm{c},{ }^{*}}$ \\ aDepartment of Neurosurgery, Shandong Provincial Hospital, Shandong University School of \\ Medicine, Jinan, Shandong, 250021 PR of China \\ bMagnetic Resonance Imaging and Spectroscopy Center, University of Kentucky, Lexington, KY \\ 40536 USA \\ 'Department of Neuroscience, University of Kentucky College of Medicine, Lexington, KY 40536 \\ USA \\ dNanning Hospital of Traditional Chinese Medicine, Nanning City, Guangxi, 530001 PR of China \\ e'Guangxi University of Chinese Medicine, Nanning City, Guangxi, 530200 PR of China
}

\section{Abstract}

Acupuncture has increasingly been used as an alternative therapy for treatment of Parkinson's disease (PD). However, the efficacy of acupunture for PD still remains unclear. The present study was designed to objectively and safely monitor anti-parkinsonian effects of electroacupuncture (EA) and brain activity in nonhuman primates modeling human PD. Six middle-aged rhesus monkeys were extensively studied by a computerized behavioral testing battery and by pharmacological MRI (phMRI) scans with specific dopaminergic drug stimulations. All animals were evaluated for behavior and phMRI responses under normal, parkinsonian, parkinsonian with EA treatment and parkinsonian after EA treatment conditions. Stable parkinsonian features were observed in all animals prior to entering the EA study and positive responses to levodopa (L-dopa) challenge were also seen in all animals. The results demonstrated that chronic EA treatments could significantly improve the movement speed and the fine motor performance time during the period of EA treatments, and the effectiveness of EA could be detected even 3 months after the EA treatment. The phMRI data revealed that chronic EA treatments could alter neuronal activity in the striatum, primary motor cortex (M1), cingulate gyrus and global pallidus externa (GPe) in the

"Corresponding author at: Department of Neuroscience, University of Kentucky, Chandler Medical Center, 48 Whitney-Hendrickson Bldg, Lexington, KY 40536-0098, USA. zzhan01@uky.edu.

Competing interests

The authors declare no actual or potential conflict of interest.

Author contributions

RZ and ZZ conceived and designed the experiments. RZ, PAH, EF, AE, YA, performed the experiments. ZZ, AHA, JY and GY analyzed and interpreted the data. RZ and ZZ wrote the manuscript. DMG, RG, and AHA helped in preparation and review of the manuscript.

Publisher's Disclaimer: This is a PDF file of an unedited manuscript that has been accepted for publication. As a service to our customers we are providing this early version of the manuscript. The manuscript will undergo copyediting, typesetting, and review of the resulting proof before it is published in its final citable form. Please note that during the production process errors may be discovered which could affect the content, and all legal disclaimers that apply to the journal pertain. 
ipsilateral hemisphere to MPTP lesions. As seen in the changes of parkinsonian features, the residual effects of phMRI responses to apomorphine (APO) challenge could also be found in the aforementioned areas. The results strongly suggest that anti-parkinsonian effects of EA can be objectively assessed, and the method used in the present study could be translated into the human clinic with some minor modifications.

\section{Keywords}

Parkinson's disease; electro-acupuncture; phMRI; behavioral; dopamine

\section{Introduction}

Acupuncture has been gaining popularity for treatment of various disorders as an alternative therapy and has been used for years for treatments from lower back pain to stroke and from osteoarthritis to Parkinson's disease (PD) (Rajendran, Thompson et al. 2001, Shulman, Wen et al. 2002, Lee, Lyu et al. 2007, Lee, Shin et al. 2008, Lei, Toosizadeh et al. 2016). Despite the widespread use of acupuncture in recent years, its efficacy remains largely unclear due to a skeptical attitude on how acupuncture works, especially within the framework of Western Medicine, methodological flaws, and a lack of high quality studies using objective outcome measures (Lee, Shin et al. 2008). To date, the clinical outcomes of acupuncture are assessed by empirical observations rather than by objective and quality analysis (Shulman, Wen et al. 2002, Lee, Shin et al. 2008, Lei, Toosizadeh et al. 2016).

The recent rapid advances in functional magnetic resonance imaging (fMRI) to map global and/or target specific bran regions have shown great promise and could be extremely helpful for acupuncture studies in human subjects if combined with other subjective measurements (Jia, Yu et al. 2012). Pharmacological MRI (phMRI) using dopaminergic drug challenges and/or fMRI techniques can provide a means to noninvasively and safely monitor brain activity in PD patients and in animals modeling human PD. In clinical studies on PD patients, acute acupuncture stimulations at GB34 can activate the putamen and the primary motor cortex, and these activations induced by acupuncture were correlated with patient selfreported improvements of finger-tapping (Chae, Lee et al. 2009). In preclinical studies of PD monkeys, phMRI has been successfully used to monitor therapeutic effects (Luan, Ding et al. 2008) and as a tool to differentiate dysfunction in basal ganglia between parkinsonian and aged monkeys (Andersen, Hardy et al. 2015). Those clinical and preclinical studies demonstrate that these imaging modalities have the possibility to untangle the underlying neural mechanisms of acupuncture.

Parkinson's disease has been well characterized by a massive loss of dopamine neurons in the substantia nigra (SN). However, clinical symptoms of PD are not manifested until the loss of dopamine neurons exceeds a critical threshold, i.e., approximately $50 \%$ of those neurons having undergone degeneration with an $80 \%$ reduction in striatal dopamine levels (Bernheimer, Birkmayer et al. 1973). Because dopamine plays a pivotal role in PD, great efforts have been made to non-invasively visualize functional changes of the central dopamine system in a living brain by using various neuroimaging techniques such as 
phMRI, especially in preclinical settings (Zhang, Andersen et al. 2000, Zhang, Andersen et al. 2006, Andersen, Hardy et al. 2015). Results from those preclinical studies in parkinsonian monkeys suggest that phMRI could be used for assessing nigrostriatal dysfunction, monitoring treatments and differentiating from age-associated motor dysfunctions (Zhang, Andersen et al. 2006, Luan, Ding et al. 2008, Andersen, Hardy et al. 2015).

The present study was designed to test the hypothesis that therapeutic effectiveness of EA can be objectively and quantitatively measured by a computerized behavioral testing battery and by phMRI scans with specific dopaminergic drug stimulations. It is worth noticing the advantages of using nonhuman primates (NHPs) in EA studies because NHPs not only share the most anatomical and physiological features with humans under normal conditions but also share behavioral and neuropathological features with humans under parkinsonian conditions (Emborg 2007, Johnston and Fox 2015). In the present study, all animals were studied under the following physiological stages: normal, hemiparkinsonian, hemiparkinsonian with EA treatments and hemiparkinsonian post EA treatments. The results from the current study will help us to better understand "where" EA-induced alternations of neural activity occur in the brain and "how" the dopamine system gets involved.

\section{Results}

\subsection{General observations}

All animals used in the study were readily adapted to the customized vertical chair designed for acupuncture procedures after $4-6$ weeks of extensive training. With similar outcomes as previously reported (Zhao, Fan et al. 2010), all animals tolerated the initial acupuncture and subsequent EA procedures well. Discomfort signs including rising of eyebrows, constant body movement and physical resistance occurred only occasionally and disappeared after properly re-adjusted the amplitude. During the initial chair and acupuncture training periods, physical conditions such as body weight, appetite, and cage activity were closely monitored in all monkeys by experienced veterinary, husbandry or research staff. No behavioral or other abnormalities were reported.

\subsection{Behavior}

As shown in Figure 1 (closed bars), significantly deceased movement speed (A), and home cage activity (B), and longer performance time of the affected hand (C) were found following MPTP administration. The movement speed and fine motor performance time were markedly improved by the chronic EA treatment (Fig. 1A \& C). The movement speed and fine motor performance time were virtually returned back to pre-MPTP levels. The cage activity was increased but did not reach statistically significant levels due to larger variance (Fig. 1B, bars with oblique lines). The fine motor performance time was still improving one month post EA treatment (Fig. 1C, bars with crossed lines). In addition, all animals responded positively to levodopa challenge evident by a $261 \%$ increase of home-cage activity measured via Actical counts. 


\section{3. phMRI scans}

Comparing with the normal stage, profound APO-induced activations were found in all measured ROIs (Figs. $2 \& 3$, Table 1). The differences between normal and post MPTP phases in response to apomorphine challenge were highly significant $(\mathrm{P}<0.001)$, especially in the caudate nucleus, putamen, M1, cingulate gyrus and GPe. In contrast, BOLD responses in the pre-motor areas and GPi were not significantly different (Table 1). Those findings were in line with previous findings (Zhang, Andersen et al. 2006) that profound APOinduced activations were seen in the striatum after animals became parkinsonian. In addition to those responses described above, APO-induced activations were also observed in the MPTP-lesioned primary motor cortex (M1) and cingulate gyrus (Fig. 3, Table 1).

The chronic EA treatment apparently altered neuronal activities in some examined areas such as the caudate nucleus, putamen, M1, cingulate gyrus and GPe in which strong APOevoked activations were seen after MPTP lesions but significantly reduced after the EA treatment. In some cases, the BOLD activations were back to near the levels seen in the normal stage (Figs. $2 \& 3$, Table 1). However, the pharmacological responses were relatively mild in the pre-motor cortex and GPi (Table 1). As shown in Figures $2 \& 3$, the most affected regions were the caudate nucleus and primary motor cortex. For example, the APOinduced activations were reduced more than 5-fold in the caudate nucleus and 4 times in primary motor cortex, respectively. Interestingly, residual effects could be found three months after the last EA treatment in the caudate nucleus, putamen, M1, cingulate gyrus regions judged by comparing the values of BOLD-activations between MPTP+EA and MPTP+PEA. A significant difference $(\mathrm{P}<0.05)$ between MPTP+EA and MPTP+PEA was also seen in the GPe as well (Table 1).

\section{Discussion}

The primary findings demonstrate that chronic EA at ST36 and LI4 can improve locomotor and fine motor functions analyzed by an objective behavioral test battery and can partially normalize APO-induced BOLD activations, which were caused by dopamine depletion in the cortico-nigro-striatal system in the hemisphere on the ipsilateral side of MPTP-lesions. In detail, the profound APO-evoked activations were significantly attenuated after the 3month chronic EA treatments, and the BOLD activations were down to near pre-MPTP (normal) levels in some ROIs. BOLD-signal changes produced by the 3-month EA treatments were accompanied by improvements in motor functions, which were objectively measured by computerized behavioral testing apparatus. The overall outcome of the present study suggests that the clinical effects of acupuncture can be objectively detected, measured and quantitatively analyzed.

The first noticeable effect associated with the chronic EA treatments was the improvement of daily physical activity including the significant increase of movement speed detected by an automatic video tracking system, and the up-trend improvement of home-cage activity measured by a computerized Actical accelerometer. Previous NHP studies have demonstrated that the movement speed and home cage activity could be good indicators for functional improvements of the central dopamine system in parkinsonian and aged animals (Grondin, Zhang et al. 2002, Maswood, Young et al. 2004, Grondin, Zhang et al. 2008, 
Luan, Ding et al. 2008). In similar settings for an acupuncture study in parkinsonian rhesus monkeys, Zhao et al. reported a greater than 3-fold increases of movement speed after 3month EA treatments at acupoint ST36 (Zhao, Fan et al. 2010). Interestingly, the movement speed measured in parkinsonian monkeys after the 3-month chronic EA treatments was comparable to what was seen in either the normal age-matched controls or to their preMPTP levels in this study. The behavioral data suggest that cumulative effects of EA could exist and may produce behavioral changes in those parkinsonian animals. In addition, the behavioral result was in agreement with our previous published studies (Grondin, Zhang et al. 2002, Grondin, Zhang et al. 2008, Luan, Ding et al. 2008). For example, steady improvements of movement speed were found in association with increases of striatal dopamine in parkinsonian monkeys with chronic intraputamenal GDNF (a dopaminergic neurotrophic factor) treatments in those aforementioned studies (Grondin, Zhang et al. 2002) and neurturin (Grondin, Zhang et al. 2008).

The automated monkey movement analysis panel (mMAP) for fine movement has been increasingly used in preclinical studies in NHPs since it was first described. Studies have demonstrated the link between performance time on the mMAP and functional status of the basal ganglia (Zhang, Andersen et al. 2000). Improved performance time on mMAP was found in all animals after chronic EA treatments in the current study which could reflect functional changes in the nigrostriatal system. Grondin et al. reported that the fine motor performance times of aged animal were significantly improved, which corresponded to a significant increase of dopamine in the caudate nucleus, putamen and substantia nigra by more than $100 \%$ (Grondin, Cass et al. 2003). In a recent review article, Leem concluded that EA could improved the motor symptoms and quality of sleep and depression in PD patients, and hypothesized that elevated nitric oxide levels may be a possible mechanism (Leem 2016). Zeng and Zhao also reported that acupuncture either manual or electroacupuncture stimulation at specific acupoints relieved some motor symptoms in patients with Parkinson's and markedly improved many nonmotor symptoms such as psychiatric disorders, sleep problems, and gastrointestinal symptoms. When it was used as an adjunct for levodopa, acupuncture improved therapeutic efficacy and reduced dosage and the occurrence of side effects of levodopa (Zeng and Zhao 2016). Therefore, it would be reasonable to assume that the behavioral improvements seen in the present study could also have resulted from functional changes in the nigrostriatal dopamine system after the 3-month EA treatments. This was also validated by another objective measurement, namely the phMRI in which the initial BOLD-activation caused by the MPTP administration was partially normalized by the 3-month EA treatments in most of the parkinsonian animals.

As mentioned above, significant reductions of APO-evoked BOLD activations were found in primary motor cortex and cingulate gyrus, the striatum and GPi on the ipsilateral side of MPTP lesions after the 3-month EA treatments. These findings were similar to observations with chronic GDNF-induced BOLD responses in parkinsonian brains (Luan, Ding et al. 2008). In the GDNF study, APO-induced activations were also significantly reduced on the side of the MPTP-lesioned putamen where the neurotrophic factor was administered. When comparing the two studies, however, the chronic EA treatments produced much broader BOLD responses than what was observed in the GDNF study, i.e., EA-induced BOLD response were seen not only in the basal ganglia but also in some cortical areas, particularly 
in the primary motor cortex. Parkinsonian animals used in the study were not treated with anti-parkinsonian medication except once for a Levodopa challenge test prior to entry into the study. Therefore, these animals could be considered as a de novo drug-naive model of human PD. In clinical fMRI studies, hypoactivations of the primary sensorimotor cortex was found in de novo Parkinson's disease (Tessa, Lucetti et al. 2012, Luo, Song et al. 2014). Therefore, it is reasonable to assume that EA induced improvements in motor functions could be through increases of brain activation, especially in the motor cortex.

Spontaneous recovery rather than chronic EA effects could reasonably be ruled out because the animal model used in the study was proven stable and has been used in various previous studies (Zhao, Fan et al. 2010), and all monkeys used in this study had expressed stable PD features for at least 12 months prior to entering the study. As we previously described (Ding, Luan et al. 2008), there are two important factors determining the probability of spontaneous recovery, namely route of administration of MPTP and age of the animals. A number of studies have demonstrated that the most spontaneous recovery reported in previous publications occurs in systemic models including intravenous, intraperitoneal, intramuscular and subcutaneous injections (Crossman, Clarke et al. 1987, Schneider, Yuwiler et al. 1987, Moratalla, Quinn et al. 1992, Albanese, Granata et al. 1993, Rose, Nomoto et al. 1993). The assumption was further supported by another study in which spontaneous behavioral recoveries were found in 4 to 6 years old young vervet monkeys after receiving multiple intramuscular injections of MPTP (Mounayar, Boulet et al. 2007). Animals used in this study were at least 15 years old with the unilateral administration of the MPTP via the carotid artery infusion, which were the key factors for maintaining stabilities of PD features seen in those animals. Overall, results of the present study support the assumption that chronic EA at ST36 and LI4 could alleviate PD symptoms through modulate brain activity, although a separate control group rather than animals used as their own controls as in this study will be needed to better confirm EA's role in improvements of PD features and alternation of brain functions in future studies.

Many of the acupoints such as LI4, ST36, GB34, LV3 that have been widely used in the treatment of PD were also used in fMRI studies (Dhond, Kettner et al. 2007). The result from some fMRI studies in PD patients suggests that therapeutic acupuncture could modulate activity in some cortical and subcortical areas. For example, Chae et al. reported that the primary motor cortex and putamen could be activated by acute acupunctures at GB34, and the acupuncture-induced BOLD activations were correlated with improvements in motor function compared with placebo-treated patients (Wu, Hsieh et al. 1999, Chae, Lee et al. 2009). These fMRI studies shed lights on how to interpret acupuncture-induced functional improvements in PD patients and whether the EA treatment can induce real changes in the parkinsonian brains. The phMRI results from the present study strongly indicate that APO-evoked BOLD activations were significantly reduced by chronic EA treatment to a level near the pre-MPTP stage in primary motor cortex, cingulate gyrus and striatum only on the ipsilateral side of MPTP lesions. This observation supports the notion that chronic EA treatments could alter brain activity and connectivity in some cortical and subcortical regions, and/or functional status of the endogenous dopaminergic neurotransmission. This assumption has been supported by a few fMRI and microdialysis studies in both animal models and humans. For example, EA could alter fMRI signals and 
dopamine release in parkinsonian rats (Chen, Wang et al. 2008, Qin, Tian et al. 2008).

However, more studies are needed to further prove the assumption.

So far, the present study demonstrates that effects of anti-parkinsonian EA treatments can be objectively and quantitatively assessed in nonhuman primates which model human PD. The methods used in this study could be practically translated into the human clinic with some minor modifications because 1) EA was performed under fully consciousness conditions, 2) the clinical MRI scanner and imaging sequences are regularly used in the clinic, and 3) all drugs and particularly the APO used in the study are already proved by the FDA for treatment of PD. Nevertheless, caution must be taken on how to interpret the animal's data for clinical use. The differences are obvious between EA treatments in PD patients and PD NHPs. First, all animals are treated equally regarding living conditions, medications and even severity of parkinsonisms. Second, among challenges in acupuncture studies in humans, the biggest issue apparently has been the lack of an adequate placebo in accessing the effectiveness of acupuncture. For obvious reasons, placebo effects are a less concerning issue in NHP studies. Nevertheless, it would be helpful for assessing EA therapeutic effects to add an additional group of animals in future studies. There are several other limitations of the present study are needed to addressed in future preclinical studies: 1) only female animals were used in the present study in concerns of reducing individual sexual variance, animals, animals handling issues and availability. Both male and female animals should be studies because sex-related differences in clinical manifestations and consequences of Parkinson's disease have been poorly explored (Farhadi, Vosoughi et al. 2017); 2) limited number of animals used in the study, which sacrificed the statistical power; and 3) all phMRI scans were performed under light anesthetized conditions due to artifacts generated from head motion. Considering the possibilities of mild effects of the chronic EA treatment, motion artifacts could significantly confound the result (Zhang, Andersen et al. 2000). Therefore light anesthesia was utilized during all phMRI scans for this study. All limitations should be carefully addressed in future studies and make this kind translational studies become more clinical relevant.

\section{Experimental procedures}

\subsection{Animals}

Six female rhesus monkeys (Macaca mulatta) ranging in age from 15-18 years old and weighing $6.2-8.6 \mathrm{~kg}$ were used in the study. All animals were housed in individual cages with water available ad libitum. Standard primate biscuits were supplemented daily with fresh fruit and vegetables. All animals underwent four phases: normal, hemiparkinsonian, hemiparkinsonian with EA treatments and hemiparkinsonian post EA treatments. In detail, each animal received the following procedures: unilateral MPTP administration, training for EA, one phMRI scan with apomorphine (APO) challenge under each phase, behavioral tests and EA treatments. Based on previously published procedures by our group, a neurotoxin MPTP $(0.12 \mathrm{mg} / \mathrm{kg})$ was unilaterally administered through the right carotid artery under sterile conditions to produce stable, moderate parkinsonian features seen in PD on the contralateral side of the body to MPTP administration. All procedures were conducted in the Laboratory Animal Facilities of the University of Kentucky, which are fully accredited by 
the Association for Assessment and Accreditation of Laboratory Animal Care International. The animals' care was supervised by experienced veterinarians and all protocols used in the study were approved by the University of Kentucky's Animal Care and Use Committee following NIH and USDA guidelines.

\subsection{Animal training for EA}

Detailed training procedures and depiction of the restraint apparatus used for EA have been previously described elsewhere (Zhao, Fan et al. 2010). In brief, animals were introduced to the specially designed vertical restraint chair used to help control the animal's movement and to have easy access to those acupoints used in the study for EA. As previously described (Zhao, Fan et al. 2010), a custom designed and manufactured vertical restraint chair with dimensions $(25 \mathrm{~cm} \times 69 \mathrm{~cm} \times 28 \mathrm{~cm})$ was made primarily of acrylic, which permits the monitoring of arm and leg movement due to its transparent feature. During each training session, animals are allowed to watch nature based television programs and are having preferred foods such as peanuts to minimize feelings of anxiety. All training sessions were taking place in the same room and were repeated 2-3 times per week until all animals were completely comfortable with the entire process.

\subsection{Behavioral evaluation}

All animals were extensively monitored and evaluated by non-biased, objective, and noninvasive methods including 1) an automated video-tracking system (EthoVision Pro, Noldus Technologies, Asheville, NC); 2) Actical accelerometers (MiniMitter, Bend, OR); 3) movement analysis panel (mMAP) for upper fine motor function.

All animals were videotaped before, during and after chronic, weekly EA treatments. The monkeys were transferred into a customized videotaping cage in the morning of the day of testing. The videotapes (DVDs) were analyzed for movement speed. The home-cage activity levels were measured by using previously published methods, i.e., an Actical accelerometer mounted on a collar worn by the animal, and activity counts per minute were recorded 24/7. Upper limb motor function was evaluated pre and post-EA using a task consisting of retrieving miniature marsh mallows from an automated receptacle chamber attached to the home cage. Motor performance was electronically recorded over 12 trials as the time (to within $10 \mathrm{~ms}$ ) for the animal to retrieve the food from a platform in the receptacle chamber (Zhang, Andersen et al. 2000).

Before entering EA studies and approximately 2 years after the MPTP treatment, all animals were challenged with levodopa, which it is the "gold" standard for treatment of PD in humans, to see if they positively responded to the dopaminergic stimulation. Each animal received an intramuscular injection of levodopa-methyl ester (75 mg Sigma Co.) and carbidopa (7.5 mg, Sigma Co.) mixed separately in sterile saline. Responsiveness to levodopa was judged by home-cage activity measured by Actical accelerometers.

\subsection{Electroacupunctural procedures}

Following our previously established standard procedures (Zhao, Fan et al. 2010), animals were introduced to acupuncture after extensive chair training. To familiarize the animal with 
the procedures, acupuncture needles were administered for 2-3 trials, each lasting approximately $30 \mathrm{~min}$. The acupoints selected for this study were based on a previous study (Zhao, Fan et al. 2010) and included: 1) HeGu (LI4), which lies halfway between the middle of metacarpal bone I and the middle of metacarpal bone II, i.e., on the dorsum of the hand, between the 1st and 2nd metacarpal bones and on the midpoint of the radial of the 2nd metacarpal bone in the hand (Fig. 1B); and 2) Zu San Li (ST36), which lies on the anteriolateral side of the leg, one finger breadth (middle finger) from the anterior crest of the tibia in the leg (Fig. 1C). The acupuncture areas were shaven and cleaned with alcohol before the needle was inserted. The stainless-steel acupuncture needles used in the study were $0.18 \mathrm{~mm}$ in diameter and $20 \mathrm{~mm}$ in length. Electro-stimulations were supplied by using Acupuncture Stimulator model 808II (AA Health Device, Tempe, AZ). Bi-directional square-wave electrical pulses $(0.2 \mathrm{~ms}$ duration, $100 \mathrm{~Hz})$ were administered for approximately 30 minutes per EA treatment. The amplitude level was $3 \mathrm{~mA}$ at the beginning and then increased gradually to 4 to $5 \mathrm{~mA}$. The animal was closely monitored by multiple video cameras. Discomfort was expressed by raised eyebrows or by withdrawal reflex or verbal expression. All animals received 3 EA treatment sessions per week for 12 weeks.

\section{5. phMRI protocols including BOLD activations}

All animals were scanned on a Siemens 3.0T TIM Trio clinical MRI system while the animals were under light general anesthesia ( $1 \%$ Isoflurane). Images were acquired using a custom designed six-channel phased array monkey head receiver coil. The BOLD-effect weighted MR images used to measure the phMRI response were acquired in an anatomically coronal plane. The image planes of the acquisition were arranged to cover the motor cortex and the basal ganglia. A segmented gradient-echo EPI sequence with TE=28 $\mathrm{ms}$ and a turbo factor of 7 was used to reduce echo train length and minimize susceptibility-related artifacts. The EPI sequence acquisition parameters are FOV $=112 \times 98 \mathrm{~mm}$ and image matrix $64 \times 56$ for an in-plane resolution of $1.75 \mathrm{~mm}$. A total of 15 contiguous slices, each $2 \mathrm{~mm}$ thick, were acquired at a rate of $15 \mathrm{~s}$ per EPI volume. The overall scan duration was 80 minutes with 128 volumes acquired prior to APO administration as a baseline and 192 after APO to track the response. Images were motion corrected and spatially smoothed using a Gaussian kernel of width $3.5 \mathrm{~mm}$. The phMRI response was calculated as the fractional signal change in $\%$ of the average of the post-APO image data relative to the pre-APO baseline. A co-registered high-resolution $(0.67 \times 0.67 \times 1 \mathrm{~mm}) \mathrm{T} 1$-weighted anatomical MRI scan was acquired in each session for spatial localization of the activation response.

\subsection{Statistical analysis}

The data were analyzed using Prism software, version 6. One-way ANOVA was followed by Bonferroni's multiple comparison tests. P-values $\$ 0.05$ were considered significant. The results are presented as the mean \pm the standard error of the mean.

\section{Acknowledgments}

This study was supported by UPPHS NIH grant NS50242 (ZZ). We also thank Drs. Feng Zhao, a neuroscientist from the Capital Medical University, and Xiaotong Fan, a Neurosurgeon from the Xuan Wu Hospital, Capital Medical University Beijing, China for their technical assistance throughout this study. 


\section{Abbreviations}

$\begin{array}{ll}\text { APO } & \text { apomorphine } \\ \text { BOLD } & \text { blood oxygen level dependent } \\ \text { EA } & \text { electroacupuncture } \\ \text { fMRI } & \text { functional magnetic resonance imaging } \\ \text { GDNF } & \text { glial cell derived neurotrophic factor } \\ \text { GPe } & \text { global pallidus externa } \\ \text { mMAP } & \text { monkey movement analysis panel } \\ \text { MPTP } & \text { 1-methyl-4-phenyl-1,2,3,6-tetrahydropyridine } \\ \text { NHP } & \text { nonhuman primate } \\ \text { PD } & \text { Parkinson's disease } \\ \text { phMRI } & \text { pharmacological MRI } \\ \text { ROI } & \text { region of interest } \\ \text { SN } & \text { substantia nigra }\end{array}$

\section{References}

Albanese A, Granata R, Gregori B, Piccardi MP, Colosimo C, Tonali P. Chronic administration of 1methyl-4-phenyl-1,2,3,6-tetrahydropyridine to monkeys: behavioural, morphological and biochemical correlates. Neuroscience. 1993; 55(3):823-832. [PubMed: 8105418]

Andersen AH, Hardy PA, Forman E, Gerhardt GA, Gash DM, Grondin RC, Zhang Z. Pharmacologic MRI (phMRI) as a tool to differentiate Parkinson's disease-related from age-related changes in basal ganglia function. Neurobiol Aging. 2015; 36(2):1174-1182. [PubMed: 25443764]

Bernheimer H, Birkmayer W, Hornykiewicz O, Jellinger K, Seitelberger F. Brain dopamine and the syndromes of Parkinson and Huntington. Clinical, morphological and neurochemical correlations. J Neurol Sci. 1973; 20(4):415-455. [PubMed: 4272516]

Chae Y, Lee H, Kim H, Kim CH, Chang DI, Kim KM, Park HJ. Parsing brain activity associated with acupuncture treatment in Parkinson's diseases. Mov Disord. 2009; 24(12):1794-1802. [PubMed: 19533753]

Chen YI, Wang FN, Nelson AJ, Xu H, Kim Y, Rosen BR, Kwong KK. Electrical stimulation modulates the amphetamine-induced hemodynamic changes: an fMRI study to compare the effect of stimulating locations and frequencies on rats. Neurosci Lett. 2008; 444(2):117-121. [PubMed: 18722508]

Crossman AR, Clarke CE, Boyce S, Robertson RG, Sambrook MA. MPTP-induced parkinsonism in the monkey: neurochemical pathology, complications of treatment and pathophysiological mechanisms. Can J Neurol Sci. 1987; 14(3 Suppl):428-435. [PubMed: 3119180]

Dhond RP, Kettner N, Napadow V. Neuroimaging acupuncture effects in the human brain. J Altern Complement Med. 2007; 13(6):603-616. [PubMed: 17718643]

Ding F, Luan L, Ai Y, Walton A, Gerhardt GA, Gash DM, Grondin R, Zhang Z. Development of a stable, early stage unilateral model of Parkinson's disease in middle-aged rhesus monkeys. Exp Neurol. 2008; 212(2):431-439. [PubMed: 18547564] 
Emborg ME. Nonhuman primate models of Parkinson's disease. Ilar j. 2007; 48(4):339-355. [PubMed: 17712221]

Farhadi F, Vosoughi K, Shahidi GA, Delbari A, Lokk J, Fereshtehnejad SM. Sexual dimorphism in Parkinson's disease: differences in clinical manifestations, quality of life and psychosocial functioning between males and females. Neuropsychiatr Dis Treat. 2017; 13:329-338. [PubMed: 28203083]

Grondin R, Cass WA, Zhang Z, Stanford JA, Gash DM, Gerhardt GA. Glial cell line-derived neurotrophic factor increases stimulus-evoked dopamine release and motor speed in aged rhesus monkeys. J Neurosci. 2003; 23(5):1974-1980. [PubMed: 12629203]

Grondin R, Zhang Z, Ai Y, Ding F, Walton AA, Surgener SP, Gerhardt GA, Gash DM. Intraputamenal infusion of exogenous neurturin protein restores motor and dopaminergic function in the globus pallidus of MPTP-lesioned rhesus monkeys. Cell Transplant. 2008; 17(4):373-381. [PubMed: 18522240]

Grondin R, Zhang Z, Yi A, Cass WA, Maswood N, Andersen AH, Elsberry DD, Klein MC, Gerhardt GA, Gash DM. Chronic, controlled GDNF infusion promotes structural and functional recovery in advanced parkinsonian monkeys. Brain. 2002; 125(Pt 10):2191-2201. [PubMed: 12244077]

Jia J, Yu Y, Deng JH, Robinson N, Bovey M, Cui YH, Liu HR, Ding W, Wu HG, Wang XM. A review of Omics research in acupuncture: the relevance and future prospects for understanding the nature of meridians and acupoints. J Ethnopharmacol. 2012; 140(3):594-603. [PubMed: 22322253]

Johnston TM, Fox SH. Symptomatic Models of Parkinson's Disease and L-DOPA-Induced Dyskinesia in Non-human Primates. Curr Top Behav Neurosci. 2015; 22:221-235. [PubMed: 25158623]

Lee MS, Shin BC, Kong JC, Ernst E. Effectiveness of acupuncture for Parkinson's disease: a systematic review. Mov Disord. 2008; 23(11):1505-1515. [PubMed: 18618661]

Lee SJ, Lyu YS, Kang HW, Sohn IC, Koo S, Kim MS, Park BR, Song JH, Kim JH. Antinociception of heterotopic electro-acupuncture mediated by the dorsolateral funiculus. Am J Chin Med. 2007; 35(2):251-264. [PubMed: 17436366]

Leem J. Acupuncture for motor symptom improvement in Parkinson's disease and the potential identification of responders to acupuncture treatment. Integr Med Res. 2016; 5(4):332-335. [PubMed: 28462136]

Lei H, Toosizadeh N, Schwenk M, Sherman S, Karp S, Sternberg E, Najafi B. A Pilot Clinical Trial to Objectively Assess the Efficacy of Electroacupuncture on Gait in Patients with Parkinson's Disease Using Body Worn Sensors. PLoS One. 2016; 11(5):e0155613. [PubMed: 27227460]

Luan L, Ding F, Ai Y, Andersen A, Hardy P, Forman E, Gerhardt GA, Gash DM, Grondin R, Zhang Z. Pharmacological MRI (phMRI) monitoring of treatment in hemiparkinsonian rhesus monkeys. Cell Transplant. 2008; 17(4):417-425. [PubMed: 18522244]

Luo C, Song W, Chen Q, Zheng Z, Chen K, Cao B, Yang J, Li J, Huang X, Gong Q, Shang HF. Reduced functional connectivity in early-stage drug-naive Parkinson's disease: a resting-state fMRI study. Neurobiol Aging. 2014; 35(2):431-441. [PubMed: 24074808]

Maswood N, Young J, Tilmont E, Zhang Z, Gash DM, Gerhardt GA, Grondin R, Roth GS, Mattison J, Lane MA, Carson RE, Cohen RM, Mouton PR, Quigley C, Mattson MP, Ingram DK. Caloric restriction increases neurotrophic factor levels and attenuates neurochemical and behavioral deficits in a primate model of Parkinson's disease. Proc Natl Acad Sci U S A. 2004; 101(52): 18171-18176. [PubMed: 15604149]

Moratalla R, Quinn B, DeLanney LE, Irwin I, Langston JW, Graybiel AM. Differential vulnerability of primate caudate-putamen and striosome-matrix dopamine systems to the neurotoxic effects of 1methyl-4-phenyl-1,2,3,6- tetrahydropyridine. Proc Natl Acad Sci U S A. 1992; 89(9):3859-3863. [PubMed: 1570304]

Mounayar S, Boulet S, Tande D, Jan C, Pessiglione M, Hirsch EC, Feger J, Savasta M, Francois C, Tremblay L. A new model to study compensatory mechanisms in MPTP-treated monkeys exhibiting recovery. Brain. 2007; 130(Pt 11):2898-2914. [PubMed: 17855373]

Qin W, Tian J, Bai L, Pan X, Yang L, Chen P, Dai J, Ai L, Zhao B, Gong Q, Wang W, von Deneen KM, Liu Y. FMRI connectivity analysis of acupuncture effects on an amygdala-associated brain network. Mol Pain. 2008; 4:55. [PubMed: 19014532] 
Rajendran PR, Thompson RE, Reich SG. The use of alternative therapies by patients with Parkinson's disease. Neurology. 2001; 57(5):790-794. [PubMed: 11552005]

Rose S, Nomoto M, Jackson EA, Gibb WR, Jaehnig P, Jenner P, Marsden CD. Age-related effects of 1methyl-4-phenyl-1,2,3,6-tetrahydropyridine treatment of common marmosets. Eur J Pharmacol. 1993; 230(2):177-185. [PubMed: 8422900]

Schneider JS, Yuwiler A, Markham CH. Selective loss of subpopulations of ventral mesencephalic dopaminergic neurons in the monkey following exposure to MPTP. Brain Res. 1987; 411(1):144150. [PubMed: 2886180]

Shulman LM, Wen X, Weiner WJ, Bateman D, Minagar A, Duncan R, Konefal J. Acupuncture therapy for the symptoms of Parkinson's disease. Mov Disord. 2002; 17(4):799-802. [PubMed: 12210879]

Tessa C, Lucetti C, Diciotti S, Paoli L, Cecchi P, Giannelli M, Baldacci F, Ginestroni A, Vignali C, Mascalchi M, Bonuccelli U. Hypoactivation of the primary sensorimotor cortex in de novo Parkinson's disease : a motor fMRI study under controlled conditions. Neuroradiology. 2012; 54(3):261-268. [PubMed: 21927866]

Wu MT, Hsieh JC, Xiong J, Yang CF, Pan HB, Chen YC, Tsai G, Rosen BR, Kwong KK. Central nervous pathway for acupuncture stimulation: localization of processing with functional MR imaging of the brain--preliminary experience. Radiology. 1999; 212(1):133-141. [PubMed: 10405732]

Zeng BY, Zhao K. Effect of Acupuncture on the Motor and Nonmotor Symptoms in Parkinson's Disease--A Review of Clinical Studies. CNS Neurosci Ther. 2016; 22(5):333-341. [PubMed: 26843036]

Zhang Z, Andersen A, Smith C, Grondin R, Gerhardt G, Gash D. Motor slowing and parkinsonian signs in aging rhesus monkeys mirror human aging. J Gerontol A Biol Sci Med Sci. 2000; 55(10):B473-480. [PubMed: 11034220]

Zhang Z, Andersen AH, Ai Y, Loveland A, Hardy PA, Gerhardt GA, Gash DM. Assessing nigrostriatal dysfunctions by pharmacological MRI in parkinsonian rhesus macaques. Neuroimage. 2006; 33(2):636-643. [PubMed: 16949305]

Zhao F, Fan X, Grondin R, Edwards R, Forman E, Moorehead J, Gerhardt G, Wang X, Zhang Z. Improved methods for electroacupuncture and electromyographic recordings in normal and parkinsonian rhesus monkeys. J Neurosci Methods. 2010; 192(2):199-206. [PubMed: 20654649] 


\section{Highlights}

1. Chronic EA treatment improves movement speed and fine motor performance time.

2. Anti-parkinsonian effects of EA can be objectively assessed.

3. The method used in the present study could be translated into the human clinic. 

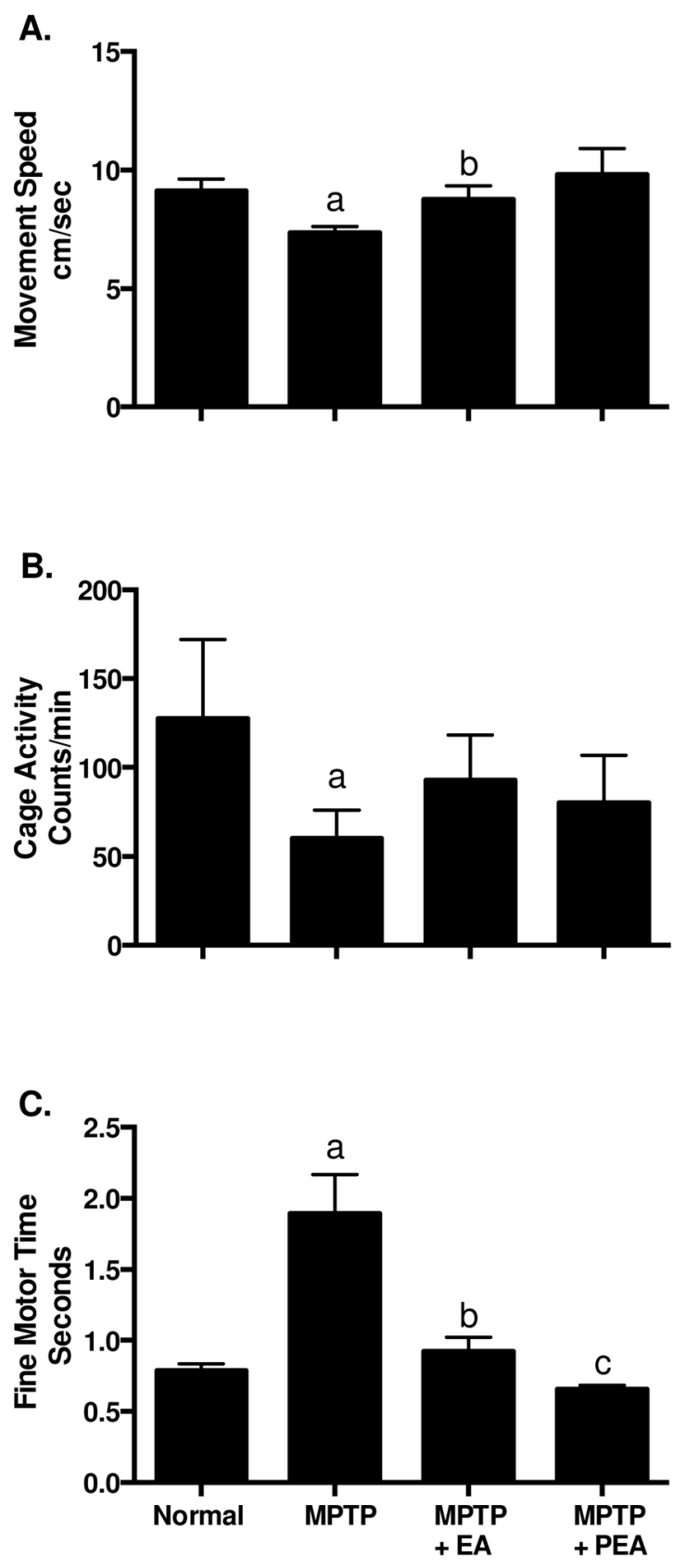

Figure 1.

Behavior changes of animals following different treatments. Movement speed (A), home cage activity $(B)$ and fine motor performance time changes $(C)$ following MPTP administration, chronic EA treatment or post EA treatment.

a, $\mathrm{P}<0.05$ compared with normal; $\mathrm{b}, \mathrm{P}<0.05$ compared with MPTP; $\mathrm{c}, \mathrm{P}<0.05$ compared with MPTP+EA. 
A. Caudate Nucleus
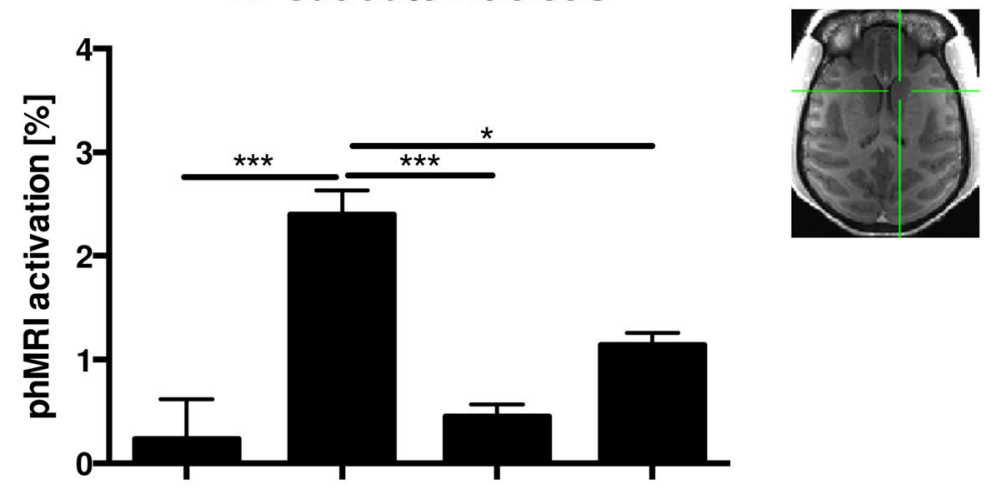

B. Putamen
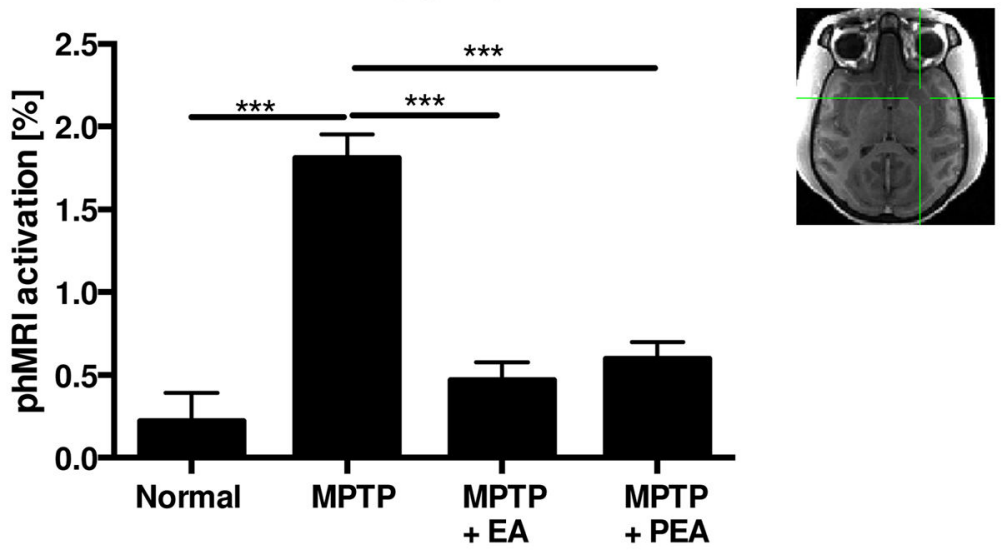

Figure 2.

phMRI activation changes of animals following different treatments. phMRI activation changes of Caudate Nucleus (A) and Putamen (B) following MPTP administration, chronic EA treatment or post EA treatment. 
Primary Motor Cortex

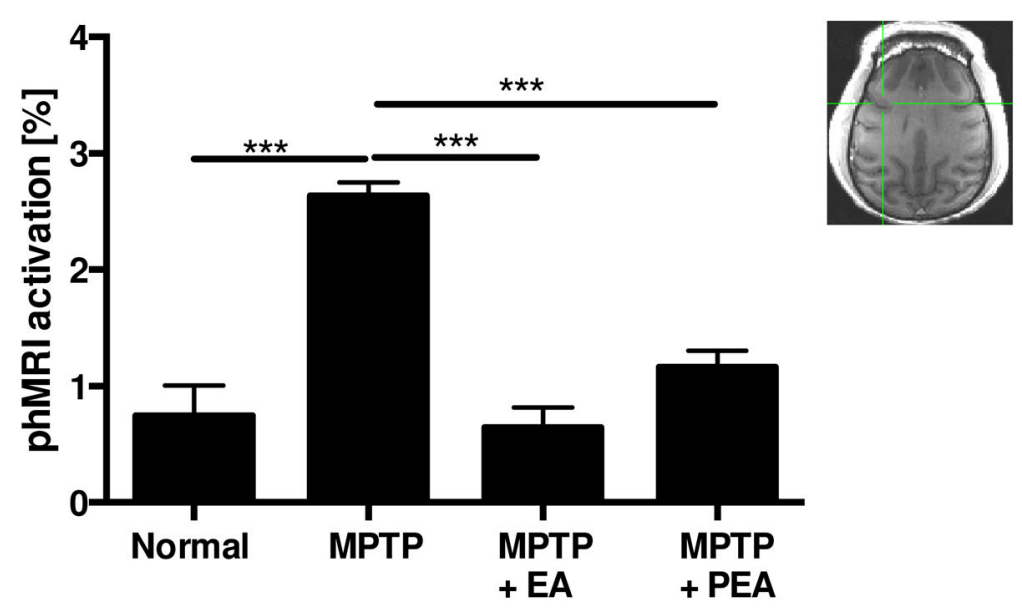

Figure 3.

phMRI activation changes of Primary Motor Cortex following MPTP administration, chronic EA treatment or post EA treatment. 


\section{Table 1}

BOLD-responses in some cortical and subcortical areas

\begin{tabular}{|c|c|c|c|}
\hline & Cingulate & GPe & GPi \\
\hline \multicolumn{4}{|l|}{ ROIs } \\
\hline Normal & $0.74 \pm 0.25$ & $-0.27 \pm 0.3$ & $-0.16 \pm 0.26$ \\
\hline MPTP & $2.16 \pm 0.09^{a}$ & $1.92 \pm 0.13^{a}$ & $0.84 \pm 0.41$ \\
\hline MPTP + EA & $0.58 \pm 0.23^{b}$ & $0.54 \pm 0.19^{d, e}$ & $0.44 \pm 0.31$ \\
\hline MPTP + PEA & $0.85 \pm 0.11^{c}$ & $0.69 \pm 0.09^{f}$ & $1.11 \pm 0.14^{d}$ \\
\hline
\end{tabular}

GPe, globus pallidus externa; GPi, globus pallidus interna; ROIs, region of interest;

${ }^{a} \mathrm{P}<0.001$ vs. normal;

$b_{\mathrm{P}<0.001 \text { vs. MPTP; }}$

$c_{\mathrm{P}<0.001 \text { vs. MPTP+EA, }}$

$d_{\mathrm{P}<0.05 \text { vs. normal; }}$

$e_{\mathrm{P}<0.01 \text { vs. MPTP; }}$

$f_{\mathrm{P}<0.05 \text { vs. MPTP+EA, }}$

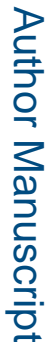

EA, elecgtroacupuncture; PEA, post elecroacupuncture. 